\title{
Outcomes of Enhanced Recovery Protocol versus Traditional Methods after Gastrointestinal Surgical Operations
}

\author{
MANSOUR M. KABAASH, M.D.*; MAHMOUD S. AHMED, M.D.*; AHMED M.A.T. EL-DABAA, M.Sc.*; \\ ABD EL-AAL A. SALEEM, M.D.** and OSAMA A. ABD EL-RHEEM, M.D.**
}

The Department of General Surgery, Faculty of Medicine, Aswan* and Sohag** Universities

\begin{abstract}
Background: Enhanced Recovery after Surgery (ERAS) program is a new set of protocols that is applied to the patient in the peri-operative period to fasten the recovery and decrease the convalescence and thus improving the surgical outcome. It is also named as fast track surgery.
\end{abstract}

Aim of Study: The aim of fast tracking is to attenuate stress response to surgical trauma in order to: Reduce morbidity, hospital stay, and costs. Enhance early and safe recovery of the patient after a surgical procedure and to allow patients to resume their normal daily activities as quick as possible.

Patients and Methods: In our study we had 50 patients underwent various gastrointestinal surgical operations. The ERAS protocol was implemented on group A with multiple pre, intra and post-operative elements, while group B was managed traditionally. Both groups were closely observed and evaluated during the post-operative period and one month after discharge.

Results: There were 6 cases that needed readmission during the first month postoperative , 2 cases in group A (8\%) and only 4 cases in group B (16\%), but this difference is insignificant. there was no obvious and significant earlier unaided mobilization in group A than group B. There was a clear difference between both groups in length of hospital stay. Patients receiving ERAS protocol stayed definitely shorter period post-operatively. There also was noticed a clear and obvious satisfaction between patients of ERAS group in the form of early mobilization, return to normal activities and pain free.

Conclusion: Fast track rehabilitation program plays an important role in the recovery of patients after gastrointestinal operations, which can accelerate the restoration of their gastrointestinal function, decrease their post-operative complications, and shorten their hospital stay time.

Key Words: ERAS - Hospital stay and costs - Gastrointestinal surgical operations.

Correspondence to: Dr. Mansour M. Kabaash, The Department of General Surgery, Faculty of Medicine, Aswan University

\section{Introduction}

ENHANCED Recovery after Surgery (ERAS) program is a new set of protocols that is applied to the patient in the peri-operative period to fasten the recovery and decrease the convalescence and thus improving the surgical outcome. It is also named as fast track surgery [1].

The Enhanced Recovery after Surgery (ERAS) is a model of care introduced in 1997 by group of general surgeons from Northern Europe led by Henrik Kehlet, then spread out in the Netherlands, United Kingdom, and Switzerland and later extended to Canada, Australasia, United States, Spain, and Latin America [2].

The concept of Enhanced Recovery after Surgery (ERAS) or multimodal surgery involves using various strategies to facilitate better conditions for surgery and recovery in an effort to achieve faster discharge from hospital and more rapid resumption of normal activities after both major and minor surgical procedures, without an increase in complications or readmissions [3].

The core of this approach was to reduce the body's reaction to surgical stress by optimizing the perioperative nutritional status, promoting analgesia without opioids and early post-operative feeding [4].

The success of ERAS programs is inextricably linked not only to the individual process measures, but also to the associated culture and adaptive management skills of the improvement team and clinician champions [5].

A recent meta-analysis showed that the application of this protocol reduced overall morbidity, 
non-surgical complications, and length of hospital stay. The ERAS pathway was also shown to be cost-effective and associated with reduced nursing workload [6].

Fast-track surgery has evolved as a result of recent evidence-based advances in the care of surgical patients. Studies investigating the effects of standard/conventional care have been performed, and show that many of the traditional approaches to surgical care, such as pre-operative bowel clearance, the use of nasogastric tubes, drains placed in cavities, enforced bed rest, and the use of graduated diets are unnecessary or even harmful [7].

Aim of the work:

The aim of fast tracking is to attenuate stress response to surgical trauma in order to:

- Reduce morbidity, hospital stay, and costs.

- Enhance early and safe recovery of the patient after a surgical procedure and to allow patients to resume their normal daily activities as quick as possible.

\section{Patients and Methods}

After approval of the Ethical Committee, this prospective comparative study has been conducted at Aswan University Hospital 2019, General Surgery Department, in the period between March 2018 till March, on 50 patients undergoing gastrointestinal surgical operations either elective or urgent operations.

After admission all patients characteristic data were obtained (age, sex, medical \& surgical history). Performing examination of the patient, laboratory investigations, routine monitoring of heart rate \& rhythm, arterial blood pressure were done.

\section{Patients have been allocated into two groups:}

Group I: Is the Enhanced Recovery After Surgery (ERAS) or study group and includes 25 patients.

Group II: Is the non ERAS or control group and includes 25 patients.

\section{Inclusion criteria:}

- Patients undergoing various gastrointestinal surgical operations.

- Age between 18 and 50 years old.

- Both genders.

\section{Exclusion criteria:}

- Age more than 50 or less than 18.

- HBG less than $12 \mathrm{~g} / \mathrm{dl}$.
- Albumin less than 3g/dl.

- Patients with clinically evident organ dysfunction.

- Patients with ongoing infections or immunosuppressive diseases.

\section{Technique:}

The study group was compared with age, sex, co-morbidity and type of operation matched control group were treated in traditional care during the same period. All patients were operated on by laparotomy.

The ERAS protocol was implemented on group A with multiple pre, intra and post-operative elements, while group B was managed traditionally. Both groups were closely observed and evaluated during the post-operative period and one month after discharge.

Patients of both groups underwent the same operations so as to obtain accurate and comparable results. The operations were appendicectomy, cholecystectomy, diverting colostomy, resection anastomosis and repair of perforated peptic ulcer.

Patients of group A (ERAS or study group) were subjected to a multimodal approach through pre, peri and postoperative components as:

Pre-operative components:

-Pre-operative counseling:

Patients should be informed with the ERAS strategy. Information discussed included:

I- What enhanced recovery involves, its core components and envisaged benefits.

II- What the patient should expect during the course of the hospital stay. This should include specifics of how ERAS is implemented locally and which modalities are employed.

III- Specific issues which may delay discharge.

IV- Clear and specific instructions should be given about mobilization and early introduction of diet:

- Curtailed fasting and pre-operative carbohydrate loading.

- Avoidance of mechanical bowel preparation.

- Deep vein thrombosis prophylaxis.

- Antibiotic prophylaxis.

Peri-operative components:

- High inspired oxygen concentration: Eighty percent (80\%) oxygen should be administered during anesthesia and then continue for at least 6 hours post-operatively. 
- Prevention of hypothermia: If the procedure is expected to last for more than an hour, then warmed intravenous fluids are used.

- Surgical approach and incision: The length of the incision should be kept as short as possible.

-Avoidance of post-operative drains, nasogastric tubes and urinary catheters: If gastric decompression is required during surgery, a nasogastric tube may be inserted temporarily and removed at the end of the procedure. However drain is not inserted unless there is extensive intraabdominal procedure or profuse bleeding and catheter only inserted according to demand of anasthetist.

- Short duration of epidural analgesia and local blocks whenever possible: This is choosen upon the preference of the operating surgeon and anaesthetist.

\section{Post-operative components:}

-Avoidance of opiates and the use of paracetamol and non-steroidal anti-inflammatory drugs.

- Early post-operative diet: Patients are allowed to have oral fluids as tolerated on the day of surgery and built up to an oral diet over the next 24 hours.

- Early post-operative mobilization: A structured mobilisation plan is followed. Patients should be helped to sit out in a chair on the evening of surgery and definitely by the first post-operative day.

- Restricted amounts of intravenous fluids: It is not possible to recommend a single point in time by which all intravenous fluid administration should be stopped. However, in the majority of patients, this should be possible by the second post-operative day, by which time adequate oral fluids should be tolerated. Excessive amounts of intravenous fluid should be avoided.

- Close monitoring and follow-up: Close monitoring of the patients early post-operative in the department and follow-up for 30 days after discharge for any post-operative complications such as wound infection, paralytic ileus, GIT discomfort, anastomotic leakage or any medical complication such as chest infection and UTI.

\section{Data management and analysis:}

The collected data was revised, coded, tabulated and introduced to a PC using Statistical package for Social Science (SPSS 25). Data was presented and suitable analysis was done according to the type of data obtained for each parameter.

\section{I- Descriptive statistics:}

1- Mean, Standard Deviation ( \pm SD) and range for parametric numerical data, while Median and Interquartile Range (IQR) for non-parametric numerical data. data.

2- Frequency and percentage of non-numerical

\section{II- Analytical statistics:}

1 - Student $t$-test was used to assess the statistical significance of the difference between two study group means.

2- Chi-Square test was used to examine the relationship between two qualitative variables.

3- Fisher's exact test was used to examine the relationship between two qualitative variables when the expected count is less than 5 in more than $20 \%$ of cells.

$p$-value: Level of significance:

$-p>0.05$ : Non significant (NS).

$-p<0.05$ : Significant (S).

- $p<0.01$ : Highly significant (HS).

\section{Results}

Group I: Cases group is the Enhanced Recovery after Surgery (ERAS) and includes 25 patients.

Group II: Control group is the non-ERAS and includes 25 patients.

\section{Demographic distribution:}

Our study was done on 50 patient from both genders divided into 2 groups. Group A contains 10 males and 15 female, Group B contains 17 male and 8 female. There is no significant difference in both groups according to demographic distribution.

Table (1): Demographic data between control and case groups.

\begin{tabular}{|c|c|c|c|c|}
\hline & \multicolumn{2}{|c|}{ Group } & \multicolumn{2}{|c|}{ Chi-Square test } \\
\hline & $\begin{array}{c}\text { Controls } \\
\mathrm{N}(\%) \\
\text { Mean } \pm \mathrm{SD}\end{array}$ & $\begin{array}{c}\text { Cases } \\
\mathrm{N}(\%) \\
\text { Mean } \pm \mathrm{SD}\end{array}$ & $p$-value & Sig \\
\hline \multicolumn{5}{|l|}{ Gender: } \\
\hline Male & $17(68 \%)$ & $15(60 \%)$ & 0.556 & NS \\
\hline Female & $8(32 \%)$ & $10(40 \%)$ & & \\
\hline Age & $34.72 \pm 9.36$ & $35 \pm 9.33$ & $0.916(\mathrm{~T})$ & NS \\
\hline
\end{tabular}

(T): Student $t$-test of significance.

Pre-operative components:

There is a obvious significant difference in both groups according pre-operative management of cases such as counseling, fasting from fluid, $\mathrm{CHO}$ loading, bowel preparation and antibiotic prophylaxis. 
Table (2): Pre-operative information between control and case groups.

\begin{tabular}{lllll}
\hline & \multicolumn{2}{c}{ Group } & \multicolumn{2}{c}{ Chi-Square test } \\
\cline { 2 - 5 } & $\begin{array}{c}\text { Controls } \\
\mathrm{N}(\%)\end{array}$ & $\begin{array}{c}\text { Cases } \\
\mathrm{N}(\%)\end{array}$ & & \\
& Mean \pm SD & Mean \pm SD & & \\
& & & \\
\hline Counseling and training & $2(8 \%)$ & $22(88 \%)$ & $<0.001 *$ & $\mathrm{~S}$ \\
Fasting from fluid & $7.28 \pm 0.98$ & $2.72 \pm 0.98$ & $<0.001 *(\mathrm{~T})$ & $\mathrm{S}$ \\
Fasting from fluid by $h r:$ & & & & \\
$\quad$ 2hrs. & $0(0 \%)$ & $16(64 \%)$ & $<0.001 *(\mathrm{~F})$ & $\mathrm{S}$ \\
$\quad$ 4hrs. & $0(0 \%)$ & $9(36 \%)$ & & \\
$\quad$ 6hrs. & $9(36 \%)$ & $0(0 \%)$ & & \\
Chrs. & $16(64 \%)$ & $0(0 \%)$ & & \\
Carbohydrate loading & $2(8 \%)$ & $22(88 \%)$ & $<0.001 *$ & $\mathrm{~S}$ \\
Bowel preparation & $12(48 \%)$ & $2(8 \%)$ & $0.002 *$ & $\mathrm{~S}$ \\
Antibiotic prophylaxis & $3(12 \%)$ & $23(92 \%)$ & $<0.001 *$ & $\mathrm{~S}$ \\
\hline
\end{tabular}

(T): Student $t$-test of significance.

(F): Monte Carlo (Fisher's Exact) test of significance.

\section{Peri-operative components:}

There is a significant difference in both groups according to use of drains and the type of anesthesia as we used general anesthesia with epidural catheter in 19 patients $(76 \%)$ of group A, while we used general anesthesia without epidural in 20 patients $(80 \%)$ of group B. However, there is no significant difference between groups in use of ryle.

Table (3): Peri-operative component between control and case groups.

\begin{tabular}{lllll}
\hline & \multicolumn{2}{c}{ Group } & $\begin{array}{c}\text { Monte Carlo } \\
\text { (Fisher's Exact) } \\
\text { test }\end{array}$ \\
\cline { 2 - 5 } & \multicolumn{1}{c}{$\begin{array}{c}\text { Controls } \\
\mathrm{N}(\%)\end{array}$} & $\begin{array}{c}\text { Cases } \\
\mathrm{N}(\%)\end{array}$ & $p$-value & Sig. \\
\hline Drains & $22(88 \%)$ & $2(8 \%)$ & $<0.001 *(\mathrm{C})$ & $\mathrm{S}$ \\
Ryle & $5(20 \%)$ & $1(4 \%)$ & 0.189 & $\mathrm{NS}$ \\
Anaesthesia: & & & & \\
General without epidural & $20(80 \%)$ & $2(8 \%)$ & $<0.001 *$ & $\mathrm{~S}$ \\
Spinal without epidural & $5(20 \%)$ & $2(8 \%)$ & & \\
Spinal with epidural & $0(0 \%)$ & $2(8 \%)$ & & \\
General with epidural & $0(0 \%)$ & $19(76 \%)$ & & \\
\hline
\end{tabular}

(C): Chi-Square test of significance.

\section{Post-operative components:}

There is highly significant difference in both groups according to post-operative components.

\section{Complications:}

Patients of group A have proven less complications than group B. Group A showed 6 complicated cases (24\%) and group B 11 cases (44\%). p -value is 0.07 which means no significance, detailed complications as in (Table 5) showed increase incidence of wound infection in group B ( 3 cases$12 \%)$ than in group A ( 2 cases- $8 \%)$ and more chest infection in group B ( 2 cases- $8 \%)$ than group A (0 case- $0 \%)$. Also there was more incidence of ileus in group B ( 2 cases- $8 \%)$ than was in group A (1 case- $4 \%$ ) also increase fecal fistula ( 2 cases- $8 \%$ ) than group A (1 case-4\%). Nausea and vomiting is the same in both groups ( 2 case- $8 \%$ ).

Table (4): Post-operative information between control and case groups.

\begin{tabular}{|c|c|c|c|c|}
\hline & \multicolumn{2}{|c|}{ Group } & \multicolumn{2}{|c|}{$\begin{array}{c}\text { Monte Carlo } \\
\text { (Fisher's Exact) } \\
\text { test }\end{array}$} \\
\hline & $\begin{array}{c}\text { Controls } \\
\mathrm{N}(\%) \\
\text { Mean } \pm \mathrm{SD}\end{array}$ & $\begin{array}{c}\text { Cases } \\
\mathrm{N}(\%) \\
\text { Mean } \pm \mathrm{SD}\end{array}$ & $p$-value & Sig. \\
\hline \multicolumn{5}{|l|}{ Pain killer: } \\
\hline Paracetamol & $3 \quad(12 \%)$ & $15(60 \%)$ & $<0.001 *(\mathrm{C})$ & $\mathrm{S}$ \\
\hline NSAIDs & $0 \quad(0 \%)$ & $10(40 \%)$ & & \\
\hline Opiates & $22(88 \%)$ & $0(0 \%)$ & & \\
\hline Pain relief & $7.08 \pm 1.15$ & $8.44 \pm 0.96$ & $<0.001 *(\mathrm{~T})$ & $S$ \\
\hline \multicolumn{5}{|c|}{ Pain relief grade: } \\
\hline Mild & $6 \quad(24 \%)$ & $1 \quad(4 \%)$ & $0.002 *$ & $S$ \\
\hline Moderate & $16(64 \%)$ & $10(40 \%)$ & & \\
\hline Severe & $3 \quad(12 \%)$ & $14(56 \%)$ & & \\
\hline \multicolumn{5}{|l|}{ Diet per oral: } \\
\hline $2 \mathrm{hrs}$ & $0 \quad(0 \%)$ & $12(48 \%)$ & $<0.001 *$ & S \\
\hline 8hrs. & $0 \quad(0 \%)$ & $13(52 \%)$ & & \\
\hline After 1 day & $12(48 \%)$ & $0(0 \%)$ & & \\
\hline 4 days & $3 \quad(12 \%)$ & $0(0 \%)$ & & \\
\hline After 4 days & $10(40 \%)$ & $0(0 \%)$ & & \\
\hline
\end{tabular}

Table (5): Complications in both control and case groups.

\begin{tabular}{|c|c|c|c|c|}
\hline & \multicolumn{2}{|c|}{ Group } & \multicolumn{2}{|c|}{$\begin{array}{c}\text { Monte Carlo } \\
\text { (Fisher's Exact) } \\
\text { test }\end{array}$} \\
\hline & $\begin{array}{c}\text { Controls } \\
\text { N }(\%) \\
\text { Mean } \pm \text { SD }\end{array}$ & $\begin{array}{c}\text { Cases } \\
\mathrm{N}(\%) \\
\text { Mean } \pm \mathrm{SD}\end{array}$ & $p$-value & Sig. \\
\hline \multicolumn{5}{|l|}{ Complications: } \\
\hline No & $14(56 \%)$ & $19(76 \%)$ & 0.737 & NS \\
\hline Nausea \& vomiting & $2(8 \%)$ & $2(8 \%)$ & & \\
\hline Wound infection & $3(12 \%)$ & $2(8 \%)$ & & \\
\hline Chest infection & $2(8 \%)$ & $0(0 \%)$ & & \\
\hline Fecal fistula & $2(8 \%)$ & $1(4 \%)$ & & \\
\hline Ileus & $2(8 \%)$ & $1(4 \%)$ & & \\
\hline
\end{tabular}

Hospital stay:

The length of hospital stay was categorized into 4 categories and comparison between both groups according to the duration of hospital stay was done.

Group A: 14 patients (56\%) have spent less than 2 days, 10 patients ( $40 \%$ ) have spent 2-4 days, 1 patient (4\%) has spent more than 4 days, no patient $(0 \%)$ has spent more than 6 days.

Group B: 4 patients (16\%) have spent less than 2 days, 5 patients (20\%) have spent 2-4 days, 5 patients $(20 \%)$ have spent more than 4 days, 11 patients (44\%) have spent more than 6 days.

$p$-value is 0.001 which is highly significant. 
Table (6): Hospital stay between control and case groups.

\begin{tabular}{|c|c|c|c|c|}
\hline & \multicolumn{2}{|c|}{ Group } & \multicolumn{2}{|c|}{$\begin{array}{c}\text { Monte Carlo } \\
\text { (Fisher's Exact) } \\
\text { test }\end{array}$} \\
\hline & $\begin{array}{c}\text { Controls } \\
N(\%) \\
\text { Mean } \pm \text { SD }\end{array}$ & $\begin{array}{c}\text { Cases } \\
\mathrm{N}(\%) \\
\text { Mean } \pm \mathrm{SD}\end{array}$ & $p$-value & Sig. \\
\hline $\begin{array}{l}\text { Hospital stay: } \\
\text { Less than } 2 \text { days } \\
\text { 2-4 days } \\
\text { More than } 4 \text { days } \\
\text { More than } 6 \text { days }\end{array}$ & $\begin{array}{l}4(16 \%) \\
5(20 \%) \\
5(20 \%) \\
11(44 \%)\end{array}$ & $\begin{array}{l}14(56 \%) \\
10(40 \%) \\
1(4 \%) \\
0(0 \%)\end{array}$ & $<0.001 *$ & S \\
\hline
\end{tabular}

\section{Patient satisfaction:}

Patient satisfaction $p$-value 0.024 and was categorized to total-mild-and poor-satisfaction according to pain control and quality of recovery in both groups $p$-value 0.014 which is significant as shown in (Table 7).

Table (7): Patient satisfaction between control and case groups.

\begin{tabular}{|c|c|c|c|c|}
\hline & \multicolumn{2}{|c|}{ Group } & \multicolumn{2}{|c|}{$\begin{array}{c}\text { Monte Carlo } \\
\text { (Fisher's Exact) } \\
\text { test }\end{array}$} \\
\hline & $\begin{array}{c}\text { Controls } \\
\mathrm{N}(\%) \\
\text { Mean } \pm \text { SD }\end{array}$ & $\begin{array}{c}\text { Cases } \\
\mathrm{N}(\%) \\
\text { Mean } \pm \text { SD }\end{array}$ & $p$-value & Sig. \\
\hline Patient satisfaction & $7.04 \pm 1.24$ & $7.88 \pm 1.3$ & $0.024 *(\mathrm{~T})$ & S \\
\hline $\begin{array}{l}\text { Patient satisfaction } \\
\text { grade: } \\
\text { Poor } \\
\text { Mild } \\
\text { Total }\end{array}$ & $\begin{array}{l}2(8 \%) \\
22(88 \%) \\
1(4 \%)\end{array}$ & $\begin{array}{l}1(4 \%) \\
15(60 \%) \\
9(36 \%)\end{array}$ & $0.014 *$ & S \\
\hline
\end{tabular}

\section{Discussion}

Patients from both groups have undergone various gastrointestinal surgical operations either elective or urgent. There was equality in number of different operations in both groups as following; appendicectomy 9 operations (18\%), cholecystectomy 8 operations $(16 \%)$, diverting colostomy 11 operations $(22 \%)$, resection anastomosis 12 operations (24\%), repair of perforated peptic 10 operations (20\%). It was intended for both groups to have the same operations in both so as to obtain accurate results in complications, hospital stay, early mobilization, patient satisfaction and readmission.

In our study there was a differences between both groups in number of complicated cases and the complications themselves. Patients of group A have proven less complications than group $\mathrm{B}$. Group A showed 6 complicated cases (24\%) and group B 11 cases (44\%). $p$-value is 0.0737 which means no significance. Detailed complications as in (Table 7) showed increased incidence of wound infection, chest infection, more incidence of ileus, post-operative nausea and vomiting also increase in fecal fistula in group B than group A.
According to other studies, Wind et al., [5] reported that pooled data from the six studies (three RCTs and three non-randomised comparative studies) showed that morbidity was significantly lower for fast-track programs (relative risk $0.54,95 \% \mathrm{CI}$ 0.42 to 0.69$)$. The absolute risk reduction of the pooled data was 0.15 (95\% CI 0.28 to 0.02$)$. No difference in mortality was found between the patient groups. Outcomes related to complications following surgery were reported by all studies.

Kuzma et al., [8] reported no statistically significant differences between groups in relation to morbidity rate and the frequency of abdominal cramps and vomiting. Petersen et al., [9] reported that there was no difference in complications between groups. The relative risk in the intentionto-treat analysis was $1.6(0.6-4.0)(p=0.39)$ and in the perprotocol analysis $1.7(0.5-5.3)(p=0.49)$ and no patients were re-admitted.

In our study there were 6 cases that needed readmission during the first month post-operative, 2 cases in group A ( $8 \%)$ and 4 cases in group B (16\%) but $p$-value is 0.667 which is insignificant. The causes of readmission were repeated vomiting, ileus, fecal fistula and wound infection.

Wind et al., [5] reported that after pooling available data from three RCts and three non-randomised comparative studies that readmission rates were not significantly different between the optimised and control groups (relative risk 1. 17, 95\% CI 0.73 to 1.86). Eight studies reported readmissions after the initial operation. Anderson et al., [10] reported that no patient was readmitted within 30 days of surgery and Recart et al., [11] reported that none of the patients required readmission to the hospital after discharge home. Readmission rates in the other six studies varied from 0 to $10 \%$ in the optimised group and 0 to $20 \%$ in the conventional group. Only one study reported a significant difference (which was in favour of optimisation) in readmission rates between the conventional and optimised groups.

Length of stay for inpatient care is quoted as an important index of efficiency and several changes in health care have been induced the past decades to limit the length of hospitalization. The fast track measures are designed to reduce medically unnecessary hospital stays, during which the patient could have been discharged on clinical grounds.

In our study there was a clear difference between both groups in this item. In group A 14 patients $(56 \%)$ have spent less than 2 days in comparison to 4 patients (16\%) in group B, while 
in group A 10 patients (40\%) have spent 2: 4 days in comparison to 5 patients $(20 \%)$ in group B. One patient (4\%) in group A have spent more than 4 days in comparison to 5 patients $(20 \%)$ in group $\mathrm{B}$ and 0 patients have spent more than 6 days in comparison to 11 patients (44\%) in group B. $p$ value is less than 0.001 which is highly significance. Therefore patients receiving ERAS protocol stay definitely shorter period post-operatively.

Wind et al., [5] reported that after pooling available data (from three RCTs and two nonrandomised comparative studies), that primary hospital stay in the optimised group was significantly lower than in the control group (weighted mean difference -1.56 days, 95 per cent Confidence Interval (CI) -2.61 to -0.50 days). Length of hospital stay was reported by 11 studies. In many cases, fast-track (optimised) patients had a shorter stay in hospital than control patients.

It has been suggested that reductions in length of stay after the start of fast-track program may relate to changes in organisation of care and not to a shorter recovery period, and that many of the beneficial outcomes attributed to fast track protocols (such as faster return of gastrointestinal function and mobilisation) are likely due to the positive goals set for patients before surgery. In this way, fast-track protocols use patients as a resource in planning and managing their own recovery and care.

Early mobilization in the post-operative period aims to mitigate the muscle loss, impaired pulmonary function and thrombo-embolic complications associated with bed rest.

In our study there was an obvious earlier unaided mobilization in group A than group B. $p$ value is 0.083 which is insignificant. Mobilization here means the first time the patient can move without help.

Outcomes relating to mobilisation were reported by four studies. Petersen et al., [9] reported that during the first six days of admission, mobilisation in the optimised group was more efficient than in the control group, and that the optimised group fulfilled the mobilisation goals to a greater extent than did the control group $(p<0.001)$. These authors further reported that physical activity (daily walking distances in metres) was found to significantly correlate with the ambulation time $(p=0.002)$. The median day of independence in Personal Activities of Daily Living (PADL) index ranks was the third postoperative day (range 1-4), in the intervention, and the fourth post-operative day (range 1-5) in the control group ( $p=0.22)$. The other three studies reported the time from surgery to mobilization to the toilet unaided. Anderson et al., [10] and Khoo et al., [12] reported that the optimized groups mobilized to the toilet significantly earlier than the conventional groups $(p=0.043$ and $p<0.001$ respectively), while Gatt et al., [13] reported no significant differences between the two groups.

Patient satisfaction is an important measure of quality of care that can contribute to a balanced evaluation of the structure, process and the outcome of the medical service. Many factors contribute to patient satisfaction, including convenience of the service, institutional structure, inter personal relationship, competence of health professionals and patients own expectations and preference.

It can be achieved by adequate pain control, early mobilization, and early resumption of normal daily activities. Outcomes relating to various patient satisfaction outcomes were reported by two studies. One study reported significant increases in patient satisfaction with pain management for the optimised patients but no significant differences in satisfaction with quality of recovery [11]. The other study did not report a difference between the two groups for satisfaction in hospital stay or happiness to be discharged from hospital [14]

In our study there was an evident differences between both groups in favour of ERAS group. $p$ value was 0.024 which is significant. Patients of ERAS group experienced more satisfaction in the form of pain free, early mobilization, resumption of normal activities and happiness on discharge.

Pre-operative education of patients is regarded as one of the crucial factors for fast-track rehabilitation. It is necessary to explain the detailed treatment plan, different stages of fast-track rehabilitation program and relevant measures for recovery for the patients in order to make them better understand the importance of fast-track rehabilitation program. Better cooperation of patients can bring better outcomes of fast track rehabilitation program [13]

In this study, the pre-operative counseling and training of 24 patient 22 patients $(88 \%)$ in group $\mathrm{A}$ and 2 patients (8\%) in group B. $p$-value was less than 0.001 which is significant. So pre-operative education of patients plays an important role in decreasing the rate of infection, decrease the hospital stay time and enhance the recovery of patients.

Fanaie et al., [15] found that early feeding in gastrointestinal seems to be safe, well tolerated 
and not associated with increased post-operative complaints including ileus and post-operative complications such as wound dehiscence, infection anastomotic leakage and mortality.

Early post-operative oral nutrition also plays an essential part in fast-track rehabilitation program. Food intake can stimulate gastrointestinal peristalsis, and early feeding during the first $24 \mathrm{~h}$ after surgery promotes the recovery of ileus. It has been illustrated that early post-operative oral nutrition attenuates catabolism and potentially decreases infectious complications. Consistent with this, early post-operative oral nutrition has been suggested as a routine procedure of abdominal surgery [13].

In our study there is a great difference between the two groups in which the time of post-operative oral nutrition differ as in group A 12 patient (48\%) started oral after 2 hours and 13 patients $(52 \%)$ started oral after 8 hrs in comparison with group B where 12 patient (48\%) started oral after one day, 3 patients (12\%) started oral after 4 days and 10 patients $(40 \%)$ started after more than 4 days. $p$ value was less than 0.001 which is significant. The early oral feeding of patients was done with less complications and great effect on enhance wound healing and decrease wound infection.

Also in this study, the limitation of use of ryle and drains as ryle is used once in group A (4\%) in comparison with group B used in 5 patients $(20 \%)$ that decreased the infectious complications. The outcome of fast-track rehabilitation program was better than that of conventional care.

As regard mortality, in this study no mortality was reported in the study group. This confirmed that ERP is not associated with increased mortality. Proske et al., [16] found that there were no significant differences in mortality between early oral feeding and delayed oral feeding after intestinal anastomosis. Other studies confirmed the reduction in mortality rate with early oral feeding.

Schwenk et al., [17] reported the overall mortality rates $(5.9 \%$ and $2.5 \%$ respectively; $p$ was not significant.

Fast track rehabilitation program can improve the symptoms of patients after various gastrointestinal operations better than conventional care, thus benefiting their surgery, anesthesia and pain management. The primary work of fast track rehabilitation program is the pre-operative education of patients to make them understand the whole plan and the aim of each stage. Therefore, it is necessary to get the cooperation from nurses, because they need to work professionally and nicely. Although there must be lots of difficulties in fast track rehabilitation program, it is an inevitable stage to test a new set of rules and guidelines.

In conclusion, fast track rehabilitation program plays an important role in the recovery of patients after gastrointestinal operations, which can accelerate the restoration of their gastrointestinal function, decrease their post-operative complications, and shorten their hospital stay time.

\section{References}

1- TAURCHINI M., DEL NAJA C. and TANCREDI A.: Enhanced Recovery After Surgery: A patient centered process. J. Vis. Surg., 4: 40-6, 2018.

2- LJUNGQVIST O., YOUNG-FADOK T. and DEMARTINES N.: The History of Enhanced Recovery After Surgery and the ERAS Society. J. Laparo. Endosc. Adv. Surg. Tech. A., 27: 860-2, 2017.

3- BÁNKY B., LAKATOS M., VARGA K., et al.: Enhanced Recovery Program in colorectal surgery. Magy Seb., 71 (1): 3-11, 2018

4- SENTURK J.C., KRISTO G., GOLD J., et al.: The Development of Enhanced Recovery After Surgery Across Surgical Specialties. J. Laparoendosc. Adv. Surg. Tech. A., 27: 863-70, 2017.

5- WIND J., POLLE S.W., FUNG KON JIN P.H., et al.: Systematic review of enhanced recovery programmes in colonic surgery. British Journal of Surgery, 93 (7): 8009, 2006.

6- ROULIN D., DONADINI A., GANDER S., et al.: Costeffectiveness of the implementation of an enhanced recovery protocol for colorectal surgery. Br. J. Surg., 100 (8): 1108-14, 2013.

7- DEMARTINES N., ROULIN D. and NAJJAR P.: Enhanced Recovery After Surgery Implementation: From Planning to Success. J. Laparo. Endosc. Adv. Surg. Tech. A., 27: 876-9, 2017.

8- KUZMA J.: Randomized clinical trial to compare the length of hospital stay and morbidity for early feeding with opioid-sparing analgesia versus traditional care after open appendectomy. Clinical Nutrition, 27 (5): 694-9, 2008.

9- PETERSEN M.K., MADSEN C., ANDERSEN N.T., et al.: Efficacy of multimodal optimisation of mobilisation and nutrition in patients undergoing hip replacement: A randomised clinical trial. Acta Anaesthesiology Scandinavia, 50: 712-7, 2006.

10- ANDERSON A.D., McNAUGHT C.E., MacFIE J., et al. Randomized clinical trial of multimodal optimization and standard perioperative surgical care. British Journal of Surgery, 90 (12): 1497-504, 2003.

11-RECART A., DUCHENE D., WHITE P.F., et al.: Efficacy and safety of fast-track recovery strategy for patients 
undergoing laparoscopic nephrectomy. Journal of Endourology, 19 (10): 1165-9, 2005.

12- KHOO C.K., VICKERY C.J., FORSYTH N., et al.: A prospective randomized controlled trial of multimodal perioperative management protocol in patients undergoing elective colorectal resection for cancer. Annals of Surgery, 245 (6): 867-72, 2007.

13- GATT M., REDDY B.S. and MAINPRIZE K.S.: Daycase stoma surgery: Is it feasible? Surgeon, 5 (3): 1437, 2007.

14- DELANEY C.P., ZUTSHI M., SENAGORE A.J., et al.: Prospective, randomised, controlled trial between a pathway controlled rehabilitation with early ambulation and diet and traditional post-operative care after laparotomy and intestinal resection. Diseases of the Colon \& Rectum, 46: 851-9, 2003.

15- FANAIE S.A. and ZIAEE S.A.: Safety of early oral feeding after gastrointestinal anastomosis: A randomized clinical trail. Med. J. Surg., 67: 185-8, 2015.

16- PROSKE J.M., RAUE W., NEUDECKER J., et al.: Fast track rehabilitation m colonic surgery: Results of a prospective trial. Ann. Chir., 130: 152-6, 2005.

17- SCHWENK W., GUNTHER N., WENDLING P., et al.: Fast track rehabilitation for elective colonic surgery in Germany-prospective observational data from a multicentre quality assurance programme. Int. J. Colorectal. Dis., 23: 93-9, 2008.

\section{نتائج تطبيق بروتوكول تحفيز الإستشفاء مقارثة بالطرق التقليدية

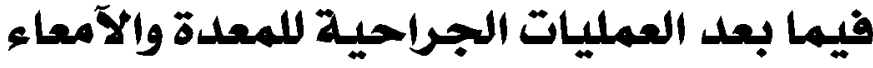

يعد برنامج تحفيز الإستشفاء ما بعد الجراحة (ERAS) مجموعة جديدة من البرتوكولات التى يتم تطبيقها على المريض فى الفترة المحيطة

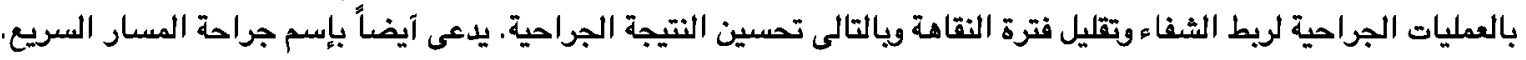

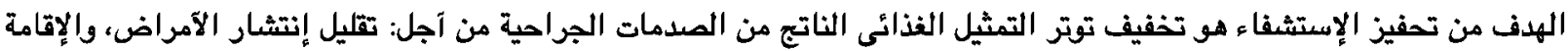

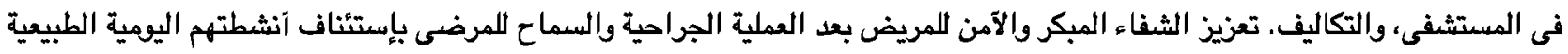
في آسرع وقت ممكن.

لقد آجريت دراستنا على • 0 مريضاً خضعوا جميعاً لعمليات جراحية متعلقة بالجهاز الهضمى. تم تتفيذ بروتوكل (ERAS) على المجموعة

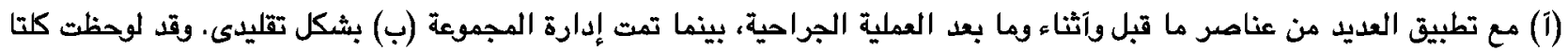

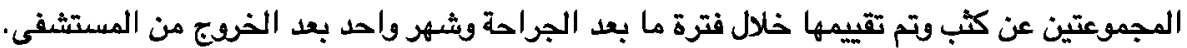

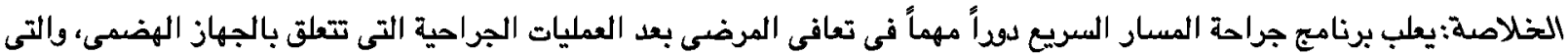

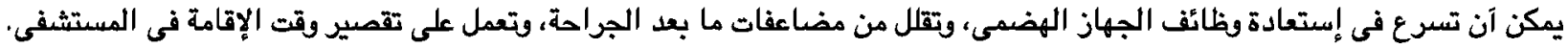

